\title{
Characterization of clinical patterns of dengue patients using an unsupervised machine learning approach
}

\author{
Gleicy Macedo Hair ${ }^{1 *}$ (D) Flávio Fonseca Nobre ${ }^{1}$ and Patrícia Brasil ${ }^{2}$
}

\begin{abstract}
Background: Despite the greater sensitivity of the new dengue clinical classification proposed by the World Health Organization (WHO) in 2009, there is a need for a better definition of warning signs and clinical progression of dengue cases. Classic statistical methods have been used to evaluate risk criteria in dengue patients, however they usually cannot access the complexity of dengue clinical profiles. We propose the use of machine learning as an alternative tool to identify the possible characteristics that could be used to develop a risk criterion for severity in dengue patients.

Method: In this study, we analyzed the clinical profiles of 523 confirmed dengue cases using self-organizing maps (SOM) and random forest algorithms to identify clusters of patients with similar patterns.

Results: We identified four natural clusters, two with features of dengue without warning signs or mild disease, one that comprises the severe dengue cases and high frequency of warning signs, and another with intermediate characteristics. Age appeared as the key variable for splitting the data into these four clusters although warning signs such as abdominal pain or tenderness, clinical fluid accumulation, mucosal bleeding, lethargy, restlessness, liver enlargement and increased hematocrit associated with a decrease in platelet counts should also be considered to evaluate severity in dengue patients.

Conclusions: These findings suggest that age must be the first characteristic to be considered in places where dengue is hyperendemic. Our results show that warning signs should be closely monitored, mainly in children. Further studies exploring these results in a longitudinal approach may help to understand the full spectrum of dengue clinical manifestations.
\end{abstract}

Keywords: Dengue, Age, Clinical classification, Warning signs, Machine learning

\section{Background}

Dengue is an acute and systemic disease caused by the dengue virus (DENV), with a broad clinical spectrum ranging from asymptomatic to severe infections. Most infections by DENV result in a mild disease known as dengue without warning signs, but a small proportion of patients develop the severe form [1].

Although the World Health Organization (WHO) issued the revised dengue guideline with a new clinical

\footnotetext{
* Correspondence: ghairbioengineer@gmail.com

${ }^{1}$ Laboratório de Engenharia em Sistemas de Saúde, Programa de Engenharia Biomédica/COPPE/UFRJ, Centro de Tecnologia - Bloco H - Sala H327, Caixa Postal (P.O. Box): 68510, Cidade Universitária, Ilha do Fundão, Rio de Janeiro, RJ 21941-972, Brazil

Full list of author information is available at the end of the article
}

classification [2], there is still debate regarding its specificity $[3,4]$. This new classification grouped the patients according to the presence or absence of warnings signs and severe dengue. Studies evaluating the new classification demonstrated a greater sensitivity when applied in endemic regions in both prospective and retrospective data, but the authors highlighted the need for a better definition of warning signs, mainly in the absence of laboratory tests $[5,6]$.

Classic statistical methods have been used to evaluate warning signs and determine risk criteria for severity in dengue patients [7-9], however, the complexity of the clinical profiles and the many overlapping levels of severity makes the disease prognosis nearly impossible to

(c) The Author(s). 2019 Open Access This article is distributed under the terms of the Creative Commons Attribution 4.0 International License (http://creativecommons.org/licenses/by/4.0/), which permits unrestricted use, distribution, and 
predict. The challenge in modeling this type of clinical data in a multifactorial disease such as dengue relies on the choice of an appropriate modeling approach that instead of providing a single predictive attribute, considers a combination of variables regardless of the data structure.

The use of unsupervised machine learning techniques is becoming popular in the medical field to reduce the dimensionality of the data and to help visualize possible patterns. The self-organizing map (SOM) is especially suitable for this task because it projects the high dimension data into a low dimension without losing the data structure [10]. Due to the increase in volume and complexity of data over the last decade, especially in the medical field, several variants of the SOM algorithm were introduced to generalize the original algorithm to handle both numerical and categorical attributes. When data are described by categorical variables or by relations between objects, a common solution is to use a measure of resemblance (i.e., a similarity or a dissimilarity measure). A general extension of this idea is a stochastic version of SOM that can be used to analyze dissimilarity data [11].

Another machine learning technique that has been successfully used in the medical field is the random forest [12]. It provides a proximity scores matrix that assesses the number of samples and the similarity/ dissimilarity matrices between them. Both matrices (similarity and dissimilarity) can be used to perform a powerful unsupervised analysis to identify patterns in the data structure.

In this study we combined random forest followed by stochastic SOM to visualize possible natural clusters associated to clinical natural patterns in dengue confirmed cases. These clusters were then reviewed for possible characteristics that could be used as risk criteria in dengue patients.

\section{Methods}

\section{Study population and eligibility criteria}

In this descriptive cross-sectional study, we analyzed retrospective data of patients with suspicion of dengue infection, assisted at the hospital of the Instituto Nacional de Infectologia Evandro Chagas/FIOCRUZ, Brazil between January 2007 and December 2013. Following the age shifting in Brazil in 2007, the Instituto Nacional de Infectologia Evandro Chagas started a project to study dengue infection in children in collaboration with three pediatric hospitals in the city. These hospitals also serve as primary care and tertiary care for dengue, therefore patients with suspicion of dengue infection and admitted into these pediatric hospitals in Rio de Janeiro RJ were also included in this analysis.
The inclusion criteria were laboratory-confirmed dengue virus (DENV) cases enrolled up to 7 days from the onset of the symptoms and followed until the outcome (cure or death), which encompassed the acute and critical phases of the disease. Patients with comorbidities and cases with more than 7 days after the onset of the symptoms at the time of admission were excluded from this analysis. Only subjects with complete data for all variables including laboratorial and clinical dengue classification were included in our analysis. All dengue cases included in this study were confirmed by at least two of the following criteria: (i) positive DENV-specific realtime reverse transcription polymerase chain reaction (RT-PCR) for any serotype (I-IV) (QIAamp Viral RNA Mini Kit, Qiagen, Hilden, Germany, following the protocol described in Lanciotti et al. [13]), (ii) at least one positive DENV-specific immunoglobulin $M(\operatorname{IgM})$ antibody in the convalescent serum compared to in the acute-phase serum or positive for qualitative IgM with dengue clinical profile during epidemic periods. Tests for detection of anti-dengue IgM were conducted using an antibody-capture enzyme-linked immunosorbent assay (PanBio, Brisbane, Australia), and/or (iii) NS1 antigen capture by using the Platelia ${ }^{\text {mi }}$ Dengue NS1 AgELISA Kit (Bio-Rad Laboratories, Marnes-La-Coquette, France) in the acute-phase serum (up to 3 days after the onset of fever), (iv) clinical-epidemiological diagnosis during epidemic periods .

\section{Data preprocessing and clinical classification}

Data were obtained from each patient's medical records. We created a categorical variable called "age group" ( $\leq 18$ years old and $>18$ years old) to better define the distribution of the variables among children and adults in the exploratory analysis. The variables age and days were normalized to reduce its variability before using to define the similarity matrix. Variables such as hematocrit and platelet increase/decrease as well as imaging data to define cavities fluid accumulation were based on at least two tests of complete blood count analyses and X-ray images, respectively. These variables were used to define some warning signs, but they were not included in this analysis.

The clinical classification was performed by trained clinicians based on the WHO guideline. It was then used to compare to the natural clusters defined by the unsupervised neural network. The classification divided the patients into three groups: dengue without warning signs, dengue with warning signs and severe dengue. Warning signs included: abdominal pain or tenderness; persistent vomiting (more than 5 times in $6 \mathrm{~h}$ or more than 3 times in $1 \mathrm{~h}$ ); clinical fluid accumulation including pleural effusion and ascites identified as a reduction of vesicular murmur or reduction of thoracic-vocal trill; 
abdominal distention or dullness decubitus, confirmed by abnormal imaging findings; mucosal hemorrhage (gastrointestinal hemorrhage and/or metrorrhagia); lethargy (alteration of consciousness and/or Glasgow score $<15)$ or irritability; and liver enlargement $(>2 \mathrm{~cm}$ below the costal margin). Laboratory findings were defined as follows: thrombocytopenia (platelet count, 50, $000 / \mathrm{mm}^{3}$ ) and hematocrit change of $20 \%$, either raised or decreased by $20 \%$ from the baseline value during the convalescent period. Severe dengue was defined by the following characteristics: (i) Plasma leakage resulting in shock or fluid accumulation with respiratory distress. Shock was defined as the presence of at least 2 of the clinical signs of hypoperfusion, with or without an associated weak pulse pressure $(\leq 20 \mathrm{mmHg})$ or hypotension for the specified age (decrease in blood arterial systolic pressure 5th percentile for age $[<\mathrm{PAS} 5]$, calculated as age [years] $2+70$ ) [14]; or (ii) severe bleeding, or (iii) severe organ involvement, e.g., severe hepatitis (aspartate aminotransferase/alanine aminotransferase levels > $1000 \mathrm{IU} / \mathrm{L}$ ); Multiple-organ dysfunction syndrome was considered when dysfunction involved 2 or more organs. Definition and clinical criteria of these signs and symptoms are better described elsewhere $[15,16]$.

\section{Unsupervised machine learning techniques \\ Random forest}

Random forest is an algorithm based on constructing a binary tree using recursive partitioning. Each binary split recursively divides the parent branch into homogeneous or near homogeneous daughter nodes (the ends of the tree). The trees are built using a two-stage randomization procedure. The first stage introduces the randomization using a bootstrap sample of the original data, and in the second, the randomization is introduced at the node level, by selecting a random subset of variables, and only those variables that keep the purity (homogeneity) of the node are kept during the split. This homogeneity is calculated by the Gini Impurity Index (Eq. 1) and it determines the purity of each node based on the relative frequency of the class in the node being evaluated.

$$
G(S)=1-\Sigma \pi_{i}^{2}
$$

where $S$ is the node being evaluated and $\pi_{\mathrm{i}}$ is the frequency of the class $\mathrm{k}$ in the node $\mathrm{S}$.

The advantage of this two-step randomization relies on the generation of decorrelate trees besides the guarantee that even those weak features are considered in the analysis [12]. An "out-of-bag" (OOB) error rate for each observation is calculated using the samples not included in the bootstrap and it is determined by majority vote across trees. Each tree is unpruned to obtain lowbias trees while bagging and random variable selection results in low correlation of the individual trees. Thus, the algorithm yields an ensemble that can achieve low bias and low variance [12].

Random forests can be used as an unsupervised technique. This approach involves the generation of a synthetic dataset to represent data without dependence. The synthetic dataset is appended to the original one, and a two-level classification variable ("original" and "synthetic") is created. Then a supervised random forest predictor is constructed to classify original from synthetic data [17]. One important output information provided by random forest predictor is a measure of the internal structure of the data (the proximity between data points). This proximity can be determined by examining the node membership of the data. Once this process is done for all trees, the proximities are normalized by dividing them by the total number of $\mathrm{t}$-trees. These scores are then stored in a proximity matrix. This matrix can be used to calculate a dissimilarity matrix by subtracting one from each of the elements, allowing a direct comparison for clustering and visualization approaches to detect data structures in high-dimensional space [17].

The Random forest algorithm was applied in an unsupervised setting to calculate the dissimilarity matrix (SOM input). All analyses were performed by using randomForest package in $\mathrm{R}$ [18].

\section{Stochastic self-organizing maps (SOM)}

SOM is a neural network that uses unsupervised competitive learning to map nonlinear statistical relationships between high-dimensional data into low dimensional grids while maintaining its original topology. Its architecture usually consists of a two-dimensional grid (input and output) with each cell in the array having a processing unit called "neuron". The neurons are connected to adjacent ones by a neighborhood function and the data points closest to each other in the input space are mapped into nearby neurons on the grid [19].

The SOM training is iterative, and it uses competitive learning where the neurons of the output layer compete to be updated. First, there is a competitive learning phase where a sample $x i$ is randomly chosen from the input data and the distance (Euclidean Distance) between the sample and all prototypes $p$ are computed [19].

The closest neuron to the input is declared the winning neuron or Best Matching Unit (BMU) $\mathrm{p}_{u}, u \in\{1, \ldots, \mathrm{U}\}$, and it can be calculated by Eq. 2 .

$$
f\left(x_{i}\right)=\arg \min _{u=1, \ldots, u}\left\|x_{i}-p_{u}\right\|
$$

where $\|$.$\| is the Euclidean distance in \Re^{d}$.

The next step consists of a cooperative phase identifying BMU's neighboring neurons using a neighborhood Gaussian function. Finally, all the prototype vectors are 
updated. It can be performed either by updating all prototypes via a weighted average (batch SOM) or in a stochastic version where the prototypes are updated mimicking a stochastic gradient descent scheme as described by the Eq. 3. The resulting grid shows the relationship between the neurons displaying the distances between input data [19].

$$
\begin{aligned}
\forall u & =1, \ldots, U \rightarrow p_{u}(t+1) \\
& =p_{u}(t)+\alpha \cdot h\left(d\left(f\left(x_{i}\right) u\right)\right)\left(x-p_{u}(t)\right)
\end{aligned}
$$

where $t$ means time, $\alpha(t)$ learning rate and $h$ is a neighborhood kernel function centered on the winner unit.

The best grid is usually chosen by checking two error measures: (1) quantization error and (2) topographic error. The topographic error or Te (Eq. 4) quantifies the continuity of the map with respect to the input space metric by counting the number of times the second-best matching (BMU2) unit of a given observation belongs to the direct neighborhood of the BMU for this observation, whereas the quantization error or Qe (Eq. 5) provides the mean distance between each vector and the cluster prototypes for $k$ clusters [10].

$$
T e=\frac{1}{n} \sum_{k=1}^{n} u\left(x_{k}\right)
$$

where $u\left(x_{k}\right)$ is 1 if the winning neuron (BMU1) and the second neuron (BMU2) are neighbors and 0 if they are not neighbors.

$$
Q e=\frac{\sum_{k=1}^{n}\left\|x_{k}-\omega_{B M U}\right\|}{n}
$$

where the mean error corresponding to the difference between the input vector $\left(x_{k}\right)$ and vector weight $(\omega \mathrm{BMU})$.

Several variants of the SOM algorithm have been introduced to overcome its limitations such as the inability of distinct types of variables and/or structure [11, 20, 21]. One of the extensions relies on the computation of a measure of similarity or dissimilarity as input data where a natural Euclidean structure is not necessarily existent, instead, the dissimilarity between the observations can be described by a dissimilarity measure $\Delta$, where $\Delta=\left(\delta_{i j}\right)_{i, j}=$ $1, \ldots, \mathrm{n}$, such that $\Delta$ is non negative $\left(\delta_{i j} \geq 0\right)$, symmetric, $\left(\delta_{i j}=\delta_{j i}\right)$ and null on the diagonal $\left(\delta_{\mathrm{ii}}=0\right) .$. In this case, the Eq. 2 cannot be carried out straightforwardly since the distances between the input data and the prototypes are not be directly computable. The solution is based on the pseudo-Euclidean framework which considers the prototypes as symbolic convex combinations of the original data [11]. In the stochastic version, it is calculated as described in the Eqs. 6 and 7 which are modification of the Eqs. 2 and 3.

$$
\begin{aligned}
& f\left(x_{i}\right)=\arg \min _{u=1, \ldots, U} D\left(\gamma_{u}\right)_{i}-\frac{1}{2} \gamma_{u}^{T} D \gamma_{u} \\
& \gamma(t+1) \leftarrow \gamma_{u}(t)+\alpha . H\left(d\left(f\left(x_{i}\right), u\right)\right)\left(1_{i}-\gamma_{u}(t)\right)
\end{aligned}
$$

where $\gamma_{\mathrm{u}}$, given $u=1, \ldots, U$ are the convex combinations of the input data and $\mathbf{1}_{i}$ is a vector with a single non-null coefficient at the $i$-th position, equal to one. Here we apply this technique to reduce the high-dimensionality and find natural patterns in the data by using the package SOMbrero in $\mathrm{R}$ [22]. This package provides the use of dissimilarity measures as input data and an ascending hierarchical clustering algorithm on the prototypes of the trained grid (superClass) for visualization of the natural clusters. The workflow of the methodologies applied is summarized in the Fig. 1.

\section{Statistical analysis}

For descriptive analyses, frequency and percentages were used for categorical variables. For continuous variables, median, range and IQR were used. Categorical variables were compared by using Chi-square test, whereas Fisher's exact test was performed when the expected table values were smaller than 5 . The difference by age and days after the onset of the symptoms among the natural clusters were compared by a one-way ANOVA analysis. Significant differences considered $p$-value $<0.05$. Statistical analyses were performed using the R statistical software R 3.5.1 [23].

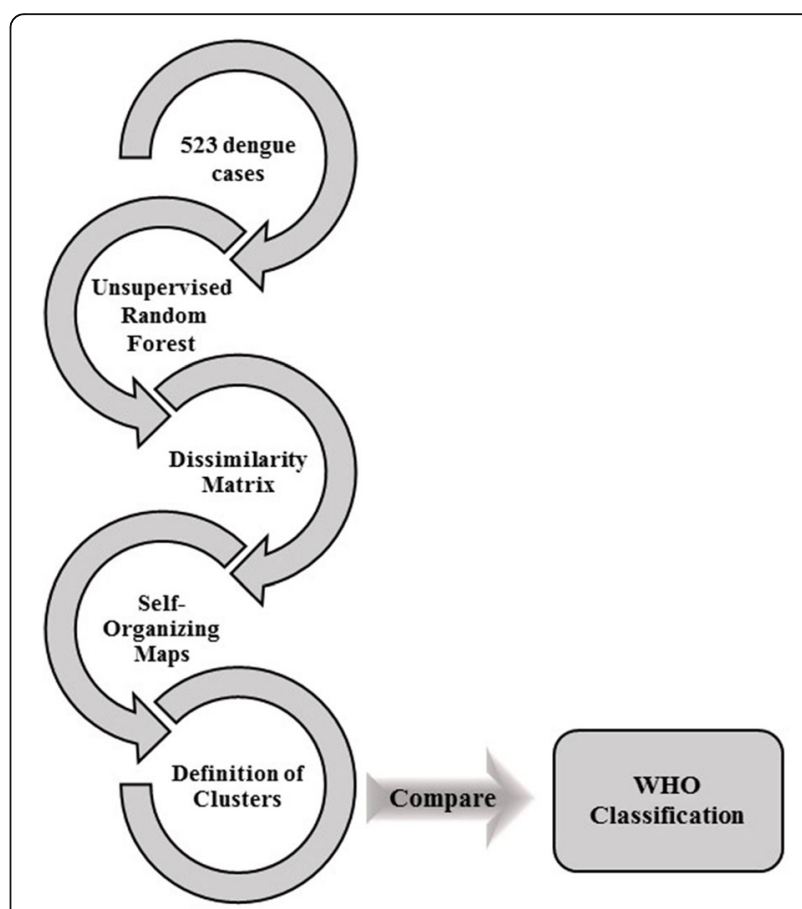

Fig. 1 Workflow 


\section{Results}

From 710 cases reported as dengue during the studied period, 93 were excluded due the lack of laboratorial data and/or missing information and/or had comorbidity, 48 had more than 7 days after the onset of the symptoms, 46 were not able to be classified due to missing information for one or more clinical variables. It resulted in 523 confirmed dengue cases that were used to identify natural patterns in the clinical profiles of patients up to 7 days of disease based on the presence of 30 different variables used for dengue clinical classification. Overall, the average age was 31 years and $49.1 \%$ were female. One hundred sixty-two $(31 \%)$ were children $(\leq 18$ years old) and 361 (69\%) were adults (> 18 years old). Dengue serotypes were identified in 183 (35\%) of the patients. The profile of the 523 patients included in this study and serotypes identified are shown in Table 1.

The more frequent clinical signs/symptoms in the children vs. adults were nausea/ vomiting (59.2\%), persistent vomiting $(56.2 \%)$, history of abdominal pain (49.4\%), abdominal pain or tenderness (48.8\%), petechiae (38.3\%), clinical fluid accumulation (pleural effusion/ascites) (31\%) and liver enlargement $>2 \mathrm{~cm}(21.6 \%)$. Adults presented more myalgia (79\%), retro-orbital eye pain (55.1\%), arthralgia (53.4\%) than children. The frequency of these signs and symptoms by age group are described in the Table 2.

The resulting dissimilarity matrix generated by unsupervised random forest was then used as input to perform the stochastic SOM algorithm. One resulting grid was selected after several trainings based on the final energy and the topographic and quantization errors. The grid with the lowest topographic error (0.05) and quantization error (0.43) was chosen (Table 3). The lowest topographic error provides the best representation of the data structure on the grid; therefore, it was prioritized.
A hierarchical clustering was then applied on the SOM prototypes to better understand and visualize the structure in the grid resulting in a dendrogram (Fig. 2), from which 4 clusters were considered as the best division. The best division was selected based on a nonparametric MANOVA described by Anderson [24]. This test is a multivariate analogue to Fisher's F-ratio and is calculated directly from any symmetric distance or dissimilarity matrix. The $P$-values are then obtained using permutations of the observations to obtain a probability associated with the null hypothesis of no differences among clusters. For 4 clusters, the results were F: 6.57 and $p$-value $<0.001$.

The numbers in each node in Fig. 2 correspond to one neuron of the SOM grid and they were grouped according to the clusters defined by the MANOVA analysis (rectangles). These clusters were then all labeled with the patient's number to obtain the classification and clinical profile from the original data (Table 4).

From 523 patients analyzed, 61 were grouped in cluster 1, 124 in cluster 2, 129 in cluster 3 and 209 in cluster 4. According to the specialist's classification (Table 4), $78.7 \%$ and $75.2 \%$ of the patients in clusters 1 and 3 were classified as Dengue without Warning Signs only (p-value: $5 \cdot 11 \mathrm{e}-14)$. Clusters 2 and 4 had $30 \cdot 7 \%$ and $39.2 \%$ of the patients classified as Dengue with warning signs (p-value: 0.001) respectively, however, cluster 4 had the highest percentage of patients classified as severe dengue, which characterize this group as more severe than the others ( $p$-value:5.55e-09). The distribution of the WHO classification by clusters is shown in the Fig. 3.

The distribution of days after the onset of symptoms between clusters was also analyzed (Fig. 4). The patients in cluster 1 were between the 1st and 3rd day of disease. Cluster 3 showed higher frequency of patients between the 2nd and 4th days. Clusters 2 and 4 showed both a

Table 1 Demographic characteristics and serotypes of the studied population according to the clinical classification

\begin{tabular}{llll}
\hline & Dengue without warning sing $(n=293)$ & Dengue with warning signs $(n=160)$ & Severe dengue $(n=70)$ \\
\hline Sex $(n$, female/male) & $(140 / 153)$ & $(72 / 88)$ & $(45 / 25)$ \\
Age (years) & Min. 0 & Min. 0 & Min. 0 \\
& Mean 31 & Mean 31 & Mean 30 \\
& Max. 79 & Max. 82 & Max. 67 \\
Age group $(n, \leq 18$ years old/> 18years old) $)$ & $(72 / 221)$ & $(61 / 99)$ & $(29 / 41)$ \\
Dengue serotype & & & $3(4.3)$ \\
DENV-1 (\%) & $20(6.8)$ & $10(6.2)$ & $2(2.9)$ \\
DENV-2 (\%) & $21(7.2)$ & $7(4.3)$ & $1(1.42)$ \\
DENV-3 (\%) & $4(1.36)$ & $4(2.5)$ & $9(12.8)$ \\
DENV-4 (\%) & $79(27)$ & $23(14.4)$ & \\
\hline
\end{tabular}


Table 2 Distribution of clinical variables by age group

\begin{tabular}{|c|c|c|c|}
\hline Variables & Abbreviations & $\begin{array}{l}\leq 18 \text { years old } \\
n=162(\%)\end{array}$ & $\begin{array}{l}>18 \text { years old } \\
n=361(\%)\end{array}$ \\
\hline Nausea/ vomiting & nau/vom & $96(59.2)$ & $142(39.3)$ \\
\hline Rash & rash & $69(42.6)$ & $177(49)$ \\
\hline History of abdominal pain & abpainhist & $80(49.4)$ & $118(32.7)$ \\
\hline Myalgia & myal & $101(62.3)$ & $285(79)$ \\
\hline Arthralgia & athral & $47(29)$ & $193(53.4)$ \\
\hline Pain behind the eyes (retro-orbital eye pain) & retpain & $58(32.1)$ & $199(55.1)$ \\
\hline+ tourniquet test & tourniq + & $5(3.1)$ & $6(1.6)$ \\
\hline Petechiae & pet & $62(38.3)$ & $96(26.6)$ \\
\hline Leukopenia & leukop & $82(50.6)$ & $175(48.5)$ \\
\hline Abdominal pain or tenderness & abpain.ws+ & 79 (48.8) & $113(31.3)$ \\
\hline Persistent vomiting & pvom.ws & $91(56.2)$ & $124(34.3)$ \\
\hline Clinical fluid accumulation (pleural effusion/ascites) & cfa.ws & $50(31)$ & $32(8.8)$ \\
\hline Mucosal bleed & bleed.ws & $53(32.7)$ & $79(21.8)$ \\
\hline Lethargy, restlessness & letha/rest.ws & $16(9.9)$ & $19(5.2)$ \\
\hline Liver enlargement $>2 \mathrm{~cm}$ & liveren.ws & $35(21.6)$ & $31(8.6)$ \\
\hline Increase hematocrit + decrease of platelet count & hto/pltws & $40(24.7)$ & $41(11.3)$ \\
\hline Dehydration & dehyd.spl ${ }^{a}$ & 37 (22.8) & $84(23.2)$ \\
\hline Edema & edema.spl & $16(9.9)$ & $23(6.4)$ \\
\hline Hypotension & hypo.spl & $15(9.2)$ & $17(4.7)$ \\
\hline Narrow pulse pressure $<20 \mathrm{mmHg}$, & $p p<20 \mathrm{mmHg} . \mathrm{spl}$ & $5(3)$ & $6(1.6)$ \\
\hline Cold clammy skin/cyanosis & coldskin.spl & $8(5)$ & $9(2.5)$ \\
\hline Rapid and weak pulse & rwp.spl & $10(6.1)$ & $11(3)$ \\
\hline Slow capillary filling & scf.spl & $16(9.9)$ & $15(4.1)$ \\
\hline Respiratory distress & respdist.spl & $15(9.2)$ & $19(5.2)$ \\
\hline Severe bleeding & severeb & $28(17.3)$ & $37(10.2)$ \\
\hline Impaired consciousness & neuro.soib & $4(2.4)$ & $18(5)$ \\
\hline Aspartate transaminase/alanine transaminase $>=1000$ & alt/ast > 1000.soi & $3(1.8)$ & $4(1.1)$ \\
\hline
\end{tabular}

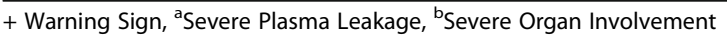

higher frequency of patients between 4th - 6th and 5th7 th days of disease respectively ( $p$-value $<0.05)$.

The age distribution between the clusters showed that clusters 1 and 2 concentrate the older patients (age range between 40 and 80 years old), cluster 3 had a higher frequency of young adults $(20-40$ years old) and cluster 4 consisted mainly of children (5-15 years old) (Fig. 5).

Table 3 SOM training features

\begin{tabular}{lll}
\hline Metric & Mean of 10 grids & Chosen grid \\
\hline Topographic Error & 0.1220 & 0.0554 \\
Quantization Error & 0.4241 & 0.4388 \\
Final Energy & 0.0120 & 0.0175 \\
\hline
\end{tabular}

A pairwise analysis was then applied to compare the proportions of each variable between the clusters. The adjusted $p$-values are shown in the Table 5 .

The variables rash, tourniquet positive test, narrow pulse pressure, cold skin, impaired consciousness and AST or ALT $>=1000$ did not have any influence on the cluster's division $(p$-values $>0 \cdot 05)$. Alternatively, the variables nausea/vomit and persistent vomiting differentiated all clusters ( $p$-values $<0.001)$. These variables were also more frequent in children $\leq 18$ years old than in adults (Table 2 ).

The patient's status (outpatient/inpatient) had also showed to be significant in the cluster's division except for clusters 1 and 3 that disclosed no difference in the distribution of this variable ( $p$-value: 0.6296).

The variables history of abdominal pain, leukopenia, and warning signs such as abdominal pain or tenderness, 


\section{Clusters Dendogram}

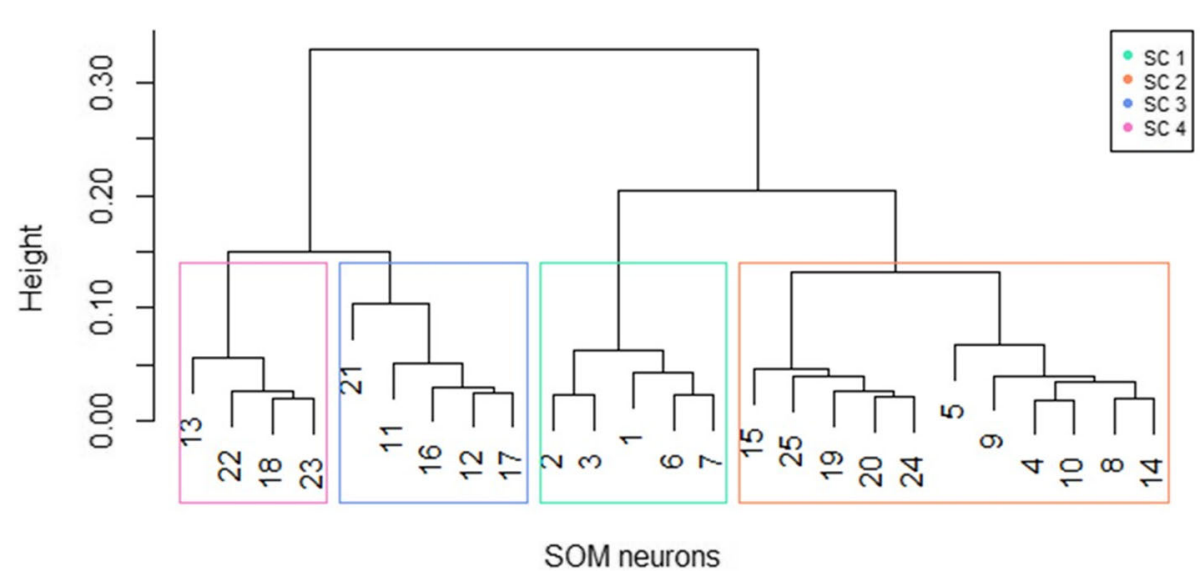

Fig. 2 Hierarchical cluster analysis applied on SOM prototypes. SC Super Cluster

clinical fluid accumulation, mucosal bleeding, lethargy, restlessness, liver enlargement and increase hematocrit associated with a decrease in platelets count were responsible for the cluster 4 definition. The variables that define shock (respiratory distress, rapid and weak pulse and slow capillary filling) were also more significant in distinguishing cluster 4 from the others. The only exception was the variable cold clammy skin/cyanosis that did not show any difference between the clusters. Other variables such as edema and hypotension were responsible only for distinguishing clusters 1 and 3 from cluster 4 but did not show any difference between clusters 1, 2 and 3 .

Whereas mucosal bleeding distinguished clusters 1 and 3 from 2 and 4, it also differentiated clusters 2 and 4 . Cluster 2 shared characteristics with cluster 4 (petechiae) and clusters 1 and 3 (myalgia, arthralgia and pain behind the eyes) which define this cluster as an intermediary profile. Dehydration was the only variable that differentiated cluster 1 from the others including cluster 3 which shares similar characteristics with cluster 1 .

\section{Discussion}

Combined unsupervised machine learning methodologies were useful to identify natural patterns in clinical dengue data, leading to the identification of four well defined clusters profiles. Two clusters (1 and 3 ) had more than $70 \%$ of the patients classified as Dengue without Warning Signs only (p-value: $5 \cdot 11 \mathrm{e}-14$ ), which could be denominated as low-risk patients. Cluster 4 had the highest percentage of patients classified as severe dengue ( $p$-value:5.55e-09) which could be labeled as high-risk group (Table 4). By using similar methodology, Faisal et al. [10] found five natural clusters that could also be clustered in two major clusters as lower risk and higher risk. However, these authors considered only laboratory data (numerical data) of patients in critical phase whereas here we included continuous and categorical variables characterized by the WHO guideline [2] to classify dengue patients in all phases (acute, critical and recovery).

The analysis of all phases of the disease suggests that, besides the rapid evolution of dengue, the transition from dengue without warning signs to dengue with warning and severe dengue happens gradually and it may be linked to the age of the patients. Patients classified as dengue without warning signs (Cluster 1) showed a higher percentage of patients in the acute phase (up to 3 days after the onset of symptoms) whereas cluster 4 , with the highest percentage of severity, had a higher percentage of patients between the critical and recovery phases (5-7 days). The same was not observed for clusters 2 and 3 . These clusters grouped the patients in the

Table 4 Clusters according to the WHO (2009) classification

\begin{tabular}{lllllllllll}
\hline Classification & Cluster 1 & $(\%)$ & Cluster 2 & $(\%)$ & Cluster 3 & $(\%)$ & Cluster 4 & $(\%)$ & ${ }^{a}$ X-squared & $P$-value \\
\hline Dengue without WS & 48 & $(78 \cdot 7)$ & 72 & $(58)$ & 97 & $(75 \cdot 2)$ & 76 & $(36 \cdot 3)$ & $64 \cdot 95$ & $5 \cdot 11 \mathrm{e}-14$ \\
Dengue with WS & 11 & $(18)$ & 38 & $(30 \cdot 7)$ & 29 & $(22 \cdot 5)$ & 82 & $(39 \cdot 2)$ & $15 \cdot 88$ & 0.001 \\
Severe Dengue & 2 & $(3 \cdot 3)$ & 14 & $(11 \cdot 3)$ & 3 & $(2 \cdot 3)$ & 51 & $(24 \cdot 5)$ & $41 \cdot 33$ & $5 \cdot 55 \mathrm{e}-09$ \\
Total & 61 & $(100)$ & 124 & $(100)$ & 129 & $(100)$ & 209 & $(100)$ & - & - \\
\hline
\end{tabular}

WS Warning signs, ${ }^{a}$ Chi-Square test: $\mathrm{HO}=$ all the proportions are equal; $\mathrm{H}_{1}=$ At least one proportion is different 


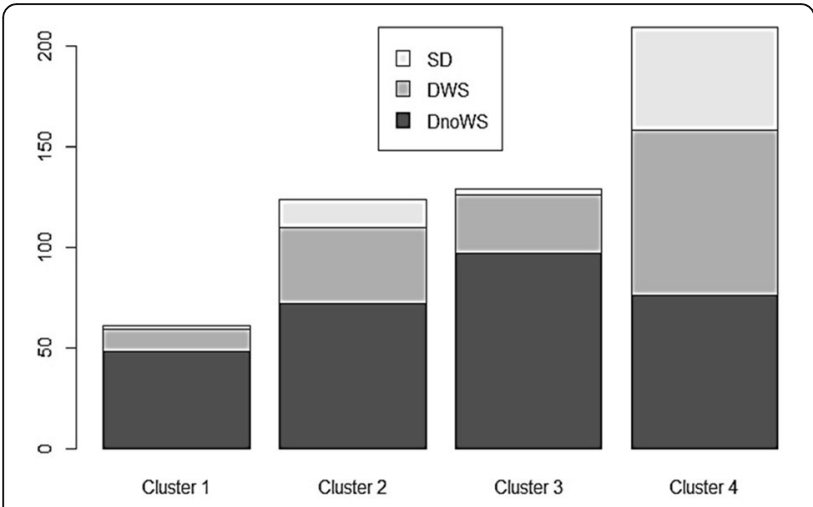

Fig. 3 Distribution of the WHO classification by cluster. SD Severe Dengue, DWS Dengue with Warning Signs, DnoWS Dengue without Warning Signs

end of the acute phase (Cluster 3) and in the beginning of the critical phase (Cluster 2) (Fig. 4). Nevertheless, these results should be further explored in future studies including age-dependence of infection and clinical presentation in a longitudinal approach.

Age appeared as the most remarkable variable in the cluster's division showing statistical significance between clusters (Fig. 5). While the low-risk cluster 1 showed a higher concentration of older patients (40-80 years old), the high-risk cluster 4 was characterized mostly by children ( $5-15$ years old). This can be explained by dengue's age shifting in 2007 in Brazil, when there was an increase of $53 \%$ of severe cases occurring in children under 15 years old, and the association of occurrence of severe dengue and hospitalization in younger patients
[26]. By simulating the force of infection of dengue based on an age stratified seroprevalence dataset, Rodriguez-Barraquer et al. [27] proposed that the conditions for the age shifting in Brazil were being set gradually and that they represent the transition from re-emergence to hyperendemicity. Our study suggests a similar association because more than $60 \%$ of patients in the high-risk cluster were younger than 10 years old (Fig. 5), so that age must be the first characteristic to be considered in dengue hyperendemic areas such as Brazil. The high-risk cluster was mainly characterized by young inpatients showing signs or symptoms of shock (Table 5), justifying the high rate of hospitalization in this cluster. Warning signs such as clinical fluid accumulation, abdominal pain, leukopenia, mucosal bleeding, lethargy, restlessness, liver enlargement and increase hematocrit (hemoconcentration) associated with the decrease of platelets (thrombocytopenia) were also crucial to discriminate this cluster from the others (Table 5). In our exploratory analysis we also found some of these symptoms more frequent in children $\leq 18$ years old than in adults (Table 2 ). Wakimoto et al. [28] confirmed that abdominal pain, bleeding, lethargy, liver enlargement, hemoconcentration with thrombocytopenia were independently associated with severe dengue in children. Our results confirm these findings and the need for monitoring these parameters in children with dengue.

There was divergent clinical presentation among the low-risk clusters. Besides these clusters sharing a higher frequency of Dengue without warning signs symptoms such as arthralgia, myalgia, and retroorbital pain, the variable dehydration was the only variable discriminating
Cluster 1

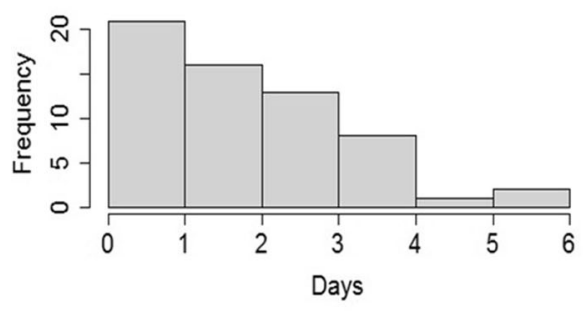

Cluster 3

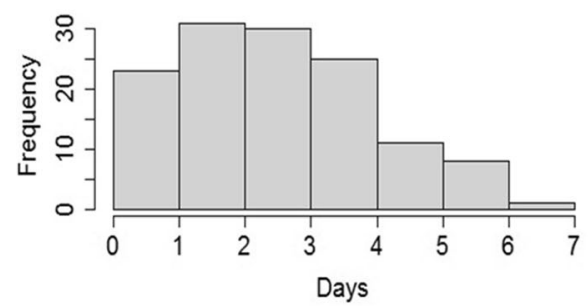

Cluster 2

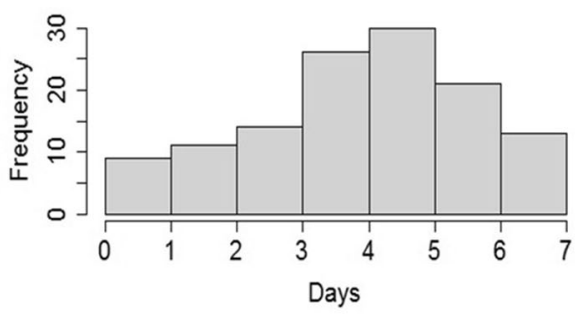

Cluster 4

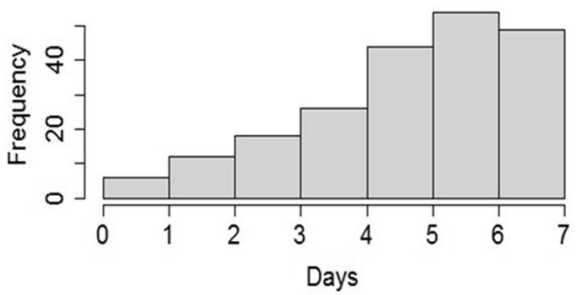

Fig. 4 Days after the onset of the symptoms according to the clusters defined by $S \mathrm{MM}^{ \pm}$. \pm There was a statistical difference between four clusters: ANOVA $F=14.43, p$-value $=4.85 e-09$ 
Cluster 1

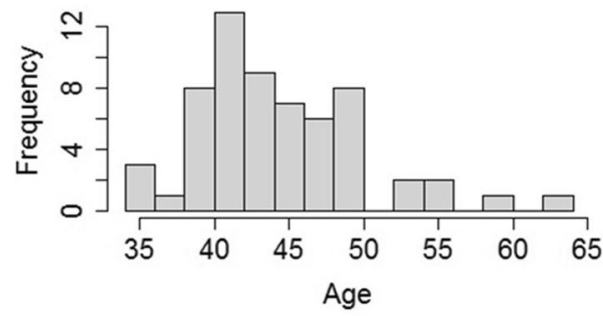

Cluster 3

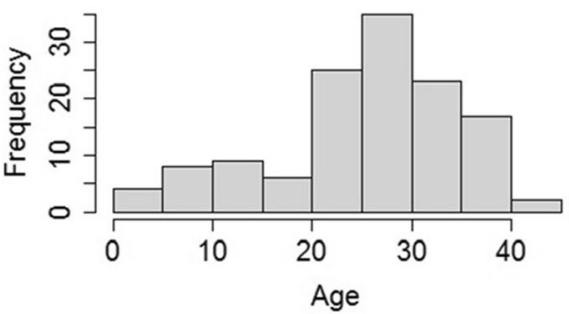

Cluster 2

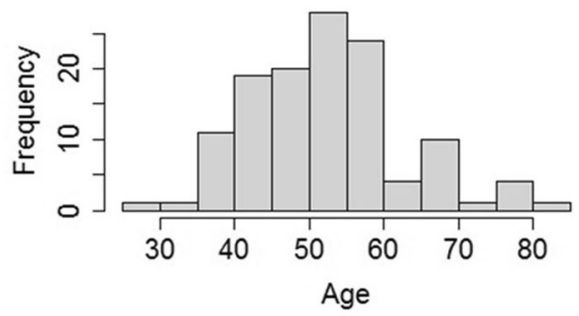

Cluster 4

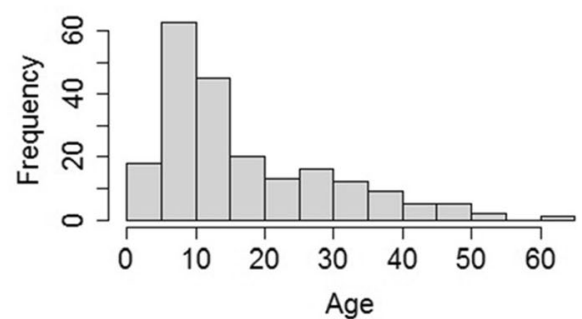

Fig. 5 Age distribution according to the clusters defined by $\mathrm{SOM}^{ \pm}$. \pm There was a statistical difference between four clusters: ANOVA $F=338.2, p$-value $=<2 e-16$

cluster 1 from the others (Table 5). In a prospective observational study in adults with median 35 years old, Thomas et al. [29] observed that dehydration and electrolyte loss was associated with severe patients with symptoms of presyncope, intense weakness, prolonged gastrointestinal symptoms, hypotension and no evidence of plasma leakage. Indeed, cluster 1 presented the lowest percentage of patients with dehydration and highest percentage of patients classified as Dengue without warning signs, indicating a good prognosis.

Cluster 2 showed intermediary characteristics, holding the second highest percentage of patients with warning signs and severe dengue but also sharing characteristics with the low-risk clusters (myalgia, arthralgia and pain behind the eyes) (Table 5). Kuo et al. [30] showed that elderly patients with dengue had significantly higher frequencies of vomiting, mucosal bleeding; higher WBC count, AST and ALT levels, and lower platelet count; when compared with their younger counterparts in critical phase. As cluster 2 was characterized by patients varying in age including children and the elderly, this cluster characteristics could represent the extreme age group of patients presenting warning signs. However, the higher percentage of children with warning signs and the lower number of elderlies included in this study makes this assumption not conclusive. Further studies are needed to characterize differences between the clinical profile in these ages.

The major limitation of this study was its cross-sectional design with retrospective data collection. Although patients were prospectively followed, clinical manifestations could have been incompletely recorded, especially among less severe cases. Therefore, the variables tourniquet positive test, narrow pulse pressure, impaired consciousness and AST/ALT levels that showed to not have any influence on the division of the clusters, need to be evaluated in more detail since the frequency of these variables were lower when compared with the others. Alternatively, the diversity of clinical profiles included in this study (ambulatory/hospitalized patients, adults/children) was an advantage, as it allowed visualization of the categorization of the full spectrum of dengue clinical manifestations. The co-circulation of several arboviruses in an endemic area such as Rio de Janeiro is a drawback that should also be considered. This study had the advantage of being conducted before the emergence of Zika and Chikungunya in the country, as they can present similar clinical manifestations.

Lastly, besides the descriptive design, this study was able to identify natural patterns in dengue clinical profile, giving insights of which clinical factors should be carefully considered in a hyperendemic area.

\section{Conclusions}

Dengue has a wide profile of clinical manifestations and the complexity of the cases with many overlapping levels of its severity has created many difficulties for the physician to predict the disease progression. Our study showed that computational techniques can be useful to identify patterns in the clinical profile of patients with dengue. Our findings suggest that age must be the first characteristic to be considered. Warning signs such as 
Table 5 Adjusted ${ }^{c} p$-values of the clinical characteristics according to each pair of clusters

\begin{tabular}{|c|c|c|c|c|c|c|}
\hline Cluster & 1vs3 & $2 v s 4$ & $2 v s 3$ & $2 \mathrm{vs} 1$ & $3 v s 4$ & 1vs4 \\
\hline nau/vom & 0.0001 & $7.9 e-05$ & 0.0001 & $8.1 \mathrm{e}-10$ & $<2 \mathrm{e}-16$ & $<2 \mathrm{e}-16$ \\
\hline rash & 1 & 1 & 1 & 0.7700 & 0.7700 & 0.3500 \\
\hline abpainhist & 1 & 7.4e-08 & 1 & 1 & $1.8 \mathrm{e}-06$ & $8.1 \mathrm{e}-06$ \\
\hline myal & 1 & 0.0061 & 0.2200 & 0.3661 & $1.6 \mathrm{e}-06$ & 0.0008 \\
\hline athral & 1 & 5.6e-05 & 1 & 0.6600 & $9.0 e-07$ & $5.5 \mathrm{e}-06$ \\
\hline retpain & 0.1557 & 0.0009 & 0.0004 & 0.2265 & $1.7 \mathrm{e}-14$ & $9.9 e-05$ \\
\hline tourniq+ & 1 & 1 & 1 & 1 & 1 & 1 \\
\hline pet & 0.1980 & 0.0899 & 0.0143 & 0.0027 & $1.7 \mathrm{e}-06$ & $3.2 \mathrm{e}-06$ \\
\hline leukop & 0.4453 & 0.0016 & 0.4453 & 1 & $4.4 \mathrm{e}-07$ & 0.0290 \\
\hline abpain.ws+ & 0.2800 & $3.4 \mathrm{e}-05$ & 0.2800 & 0.0480 & 4.6e-09 & $2.9 \mathrm{e}-08$ \\
\hline pvom.ws & 0.0008 & $5.3 e-06$ & 0.0012 & $1.1 \mathrm{e}-07$ & 7.6e-16 & $<2 \mathrm{e}-16$ \\
\hline cfa.ws & - & $1.2 \mathrm{e}-07$ & 0.0110 & 0.0730 & $4.9 e-13$ & $4.8 \mathrm{e}-07$ \\
\hline bleed.ws & 0.9560 & 0.0094 & 0.1367 & 0.4494 & $6 e-07$ & 0.0013 \\
\hline letha/rest.ws & 1 & 0.3007 & 0.1439 & 0.3007 & 0.0017 & 0.0401 \\
\hline liveren.ws & 1 & 0.0003 & 0.0338 & 0.1060 & $1.3 e-08$ & $8.2 \mathrm{e}-05$ \\
\hline hto/plt.ws & 0.7007 & 0.0005 & 0.0005 & 0.0713 & $3.0 \mathrm{e}-11$ & $2.8 \mathrm{e}-05$ \\
\hline dehyd.spl ${ }^{a}$ & 0.0070 & 0.6579 & 0.9825 & 0.0061 & 0.6579 & 0.0003 \\
\hline edema.spl & 0.5637 & 0.2934 & 0.3869 & 0.3007 & 0.0074 & 0.0271 \\
\hline hypo.spl & 1 & 0.4700 & 0.1440 & 0.3010 & 0.0050 & 0.072 \\
\hline $\mathrm{pp}<20$ mmHg.spl & - & 1 & 0.4800 & 0.9700 & 0.4400 & 0.9700 \\
\hline coldskin.spl & 1 & 0.0910 & 1 & 1 & 0.0910 & 0.2660 \\
\hline rwp.spl & 1 & 0.0128 & 1 & 1 & 0.0036 & 0.0766 \\
\hline scf.spl & 1 & 0.0006 & 1 & 1 & 0.0005 & 0.0178 \\
\hline respdist.spl & 1 & 0.0400 & 0.5924 & 0.5924 & 0.0008 & 0.0271 \\
\hline severeb & 1 & 0.0156 & 1 & 1 & 0.0004 & 0.1417 \\
\hline neuro.soib ${ }^{\mathrm{b}}$ & 1 & 1 & 0.7300 & 1 & 1 & 1 \\
\hline alt/ast > 1000.soi & 1 & 1 & 1 & 1 & 1 & 1 \\
\hline inpatient & 0.6296 & $1.5 \mathrm{e}-06$ & 7.6e-05 & 0.0003 & $<2 \mathrm{e}-16$ & $1.6 \mathrm{e}-14$ \\
\hline
\end{tabular}

+Warning Sign, ${ }^{\mathrm{a}}$ Severe Plasma Leakage, ${ }^{\mathrm{b}}$ Severe Organ Involvement, ${ }^{\mathrm{C} C h i-S q u a r e}$ test: $\mathrm{H} 0=$ all the proportions are equal; $\mathrm{H} 1=$ At least one proportion is different. 'Based on Holm [25]

abdominal pain or tenderness, clinical fluid accumulation, mucosal bleeding, lethargy, restlessness, liver enlargement and increase hematocrit should be closely monitored, mainly in children. Further studies exploring these results in a longitudinal approach could be useful to create models to help clinicians and pediatricians to predict severity in dengue infection, mainly in areas where others arbovirus also circulates.

\section{Abbreviations}

abpain.ws: Abdominal pain or tenderness; abpainhist: History of abdominal pain; alt/ast > 1000.soi: Aspartate transaminase/alanine transaminase > = 1000; athral: Arthralgia; bleed.ws: Mucosal bleed; BMU: Best Matching Unit; cfa.ws: Clinical fluid accumulation (pleural effusion/ascites); coldskin.spl: Cold clammy skin/cyanosis; dehyd.spl: Dehydration; DENV: Dengue Virus; edema.spl: Edema; hto/plt.ws: Increase hematocrit + decrease of platelet count; hypo.spl: Hypotension; IgM: Immunoglobulin M; letha/ rest.ws: Lethargy, restlessness; leukop: Leukopenia; liveren.ws: Liver enlargement > 2 cm; myal: Myalgia; nau/vom: Nausea/ vomiting; neuro.soi: Impaired consciousness; NS1: Non-structural protein 1; pet: Petechiae; PP: Pulse pressure; pp. $<20 \mathrm{mmHg}$.spl: Narrow pulse pressure < $20 \mathrm{mmHg}$; pvom.ws: Persistent vomiting; rash: Rash; respdist.spl: Respiratory distress; retpain: Pain behind the eyes (retro-orbital eye pain); RT-PCR: Reverse transcription polymerase chain reaction; rwp.spl: Rapid and weak pulse; scf.spl: Slow capillary filling; severeb: Severe bleeding; SOI: Severe organ involvement; SOM: Self-organizing maps; SPL: Severe Plasma Leakage; tourniq+: + tourniquet test; WHO: World Health Organization; WS: Warning signs

\section{Acknowledgments}

We would like to acknowledge the clinicians and technicians for the data collection/review; without whose contribution this research couldn't have gone so far.

\section{Authors' contributions}

GMH, FFN and PB designed the study. GMH and FFN analyzed the data. All authors wrote and edited initial drafts and reviewed the final draft. All authors read and approved the final manuscript. 


\section{Funding}

PB is supported by the Consellho Nacional de Desenvolvimento e Pesquisa (CNPq/307282/2017-1) and Fundação de Amparo à Pesquisa do Estado do Rio de Janeiro (FAPERJ/ E_26/202.862/2018 CNE) and FFN has a grant from CNPq (CNPQ/302258/2017-5). This study was also financed in part by the Coordenação de Aperfeiçoamento de Pessoal de Nível Superior - Brasil (CAPES) - Finance Code 001F. The funders had no role in this study design, data collection, analysis, interpretation, or writing of the report. The corresponding author had full access to all data in the study and had final responsibility for the decision to submit for publication.

\section{Availability of data and materials}

The datasets generated and/or analyzed during the current study are not publicly available due sensitivity of the data, but the scripts used for the analysis are available from the corresponding author on reasonable request.

\section{Ethics approval and consent to participate}

This study is based on data routinely collected in the hospital of the Instituto Nacional de Infectologia Evandro Chagas/FIOCRUZ and three pediatric hospitals in the city. The study was reviewed and approved by the Institutional Review Board (IRB) of Fundação Oswaldo Cruz (Protocol n’: 1532.0.009.000-1 161/08, CAAE: 0026.0.009.000-07) and Secretaria Municipal de Saúde e Defesa Civil do Rio de Janeiro, RJ (33/09 CAAE: 00290314011-09). Informed consent was waived since the data were anonymized and de-identified prior to analysis to ensure patient's information confidentiality.

\section{Consent for publication}

Not applicable.

\section{Competing interests}

The authors declare that they have no competing interests.

\section{Author details}

${ }^{1}$ Laboratório de Engenharia em Sistemas de Saúde, Programa de Engenharia Biomédica/COPPE/UFRJ, Centro de Tecnologia - Bloco H - Sala H327, Caixa Postal (P.O. Box): 68510, Cidade Universitária, Ilha do Fundão, Rio de Janeiro, RJ 21941-972, Brazil. ${ }^{2}$ Acute Febrile IIInesses Laboratory, Evandro Chagas National Institute of Infectious Diseases; Oswaldo Cruz Foundation (Fiocruz), Rio de Janeiro, RJ, Brazil.

Received: 9 May 2018 Accepted: 11 July 2019

\section{Published online: 22 July 2019}

\section{References}

1. Simmons CP, Farrar JJ, Nguyen van VC, Wills B. Dengue. N Engl J Med. 2012; 366:1423-32.

2. World Health Organization. Dengue: guidelines for diagnosis, treatment, prevention and control. Geneva; 2009. https://apps.who.int/iris/bitstream/ handle/10665/44188/9789241547871_eng.pdf;jsessionid=517670732D36D32 F6945CE52E76A3258? sequence $=1$. Accessed 10 June 2013

3. Horstick O, Farrar J, Lum L, Martinez E, San Martin JL, Ehrenberg J, et al. Reviewing the development, evidence base, and application of the revised dengue case classification. Pathog Glob Health. 2012;106:94-101.

4. Halstead SB. Dengue: the syndromic basis to pathogenesis research. Inutility of the 2009 WHO case definition. Am J Trop Med Hyg. 2013;88:212-5.

5. Barniol J, Gaczkowski R, Barbato EV, da Cunha RV, Salgado D, Martínez E, et al. Usefulness and applicability of the revised dengue case classification by disease: multi-center study in 18 countries. BMC Infect Dis. 2011;11:106.

6. Macedo GA, Gonin MLC, Pone SM, Cruz OG, Nobre FF, Brasil P. Sensitivity and specificity of the World Health Organization dengue classification schemes for severe dengue assessment in children in Rio de Janeiro. PLoS One. 2014;9:e96314.

7. Fernández E, Smieja M, Walter SD, Loeb M. A retrospective cohort study to predict severe dengue in Honduran patients. BMC Infect Dis. 2017;17:676.

8. Lee I-K, Liu J-W, Chen Y-H, Chen Y-C, Tsai C-Y, Huang S-Y, et al. Development of a simple clinical risk score for early prediction of severe dengue in adult patients. PLoS One. 2016;11. https://doi.org/10.1371/journal. pone.0154772.

9. Suwarto S, Hidayat MJ, Widjaya B. Dengue score as a diagnostic predictor for pleural effusion and/or ascites: external validation and clinical application. BMC Infect Dis. 2018;18:90.
10. Faisal T, Taib MN, Ibrahim F. Reexamination of risk criteria in dengue patients using the self-organizing map. Med Biol Eng Comput. 2010;48: 293-301.

11. Olteanu M, Villa-Vialaneix N. On-line relational and multiple relational SOM. Neurocomputing. 2015;147:15-30.

12. Breiman L. Random forests. Mach Learn. 2001;45:5-32.

13. Lanciotti RS, Calisher CH, Gubler DJ, Chang GJ, Vorndam AV. Rapid detection and typing of dengue viruses from clinical samples by using reverse transcriptase-polymerase chain reaction. J Clin Microbiol. 1992;30: 545-51.

14. Goldstein B, Giroir B, Randolph A. International consensus conference on pediatric sepsis. International pediatric sepsis consensus conference: definitions for sepsis and organ dysfunction in pediatrics. Pediatr Crit Care Med. 2005;6:2-8.

15. WHO | Dengue: guidelines for diagnosis, treatment, prevention and control. https://apps.who.int/iris/bitstream/handle/10665/44188/9789241547871_ eng.pdf;jsessionid=517670732D36D32F6945CE52E76A3258? sequence $=1$. Accessed 29 May 2019.

16. PAHO. Dengue: guidelines for patient care in the Region of the Americas. 2nd ed; 2016. http://iris.paho.org/xmlui/bitstream/handle/123456789/31207/ 9789275118900-eng.pdf? sequence=1\&isAllowed=y. Accessed 29 May 2019.

17. Afanador NL, Smolinska A, Tran T, Blanchet L. Unsupervised random forest: a tutorial with case studies. J Chemom. 2016;30:231.

18. Liaw A, Wiener M. Classification and regression by randomForest. R News. 2002;2:18-22.

19. Kohonen T. The self-organizing map. Proc IEEE. 1990;78:1464-80.

20. Chen N, Marques NC. An Extension of Self-organizing Maps to Categorical Data. In: Bento C, Cardoso A, Dias G, editors. Progress in Artificial Intelligence. Berlin Heidelberg: Springer; 2005. p. 304-13.

21. Hsu C-C. Generalizing self-organizing map for categorical data. IEEE Trans Neural Netw. 2006;17:294-304.

22. Olteanu M, Villa-Villaneix N. Using SOMbrero for clustering and visualizing graphs. J French Stat Soc. 2015;156:95-119.

23. R Core Team. R: A language and environment for statisticalcomputing. Vienna: R Foundation for Statistical Computing; 2018.URL http://www.Rproject.org/.

24. Anderson MJ. A new method for non-parametric multivariate analysis of variance. Austral Ecol. 2001;26:32-46.

25. Holm S. A simple sequentially rejective multiple test procedure. Scand J Stat. 1979;6:65-70.

26. Teixeira MG, Costa MCN, Coelho G, Barreto ML. Recent shift in age pattern of dengue hemorrhagic fever, Brazil. Emerg Infect Dis. 2008;14:1663.

27. Rodriguez-Barraquer I, Cordeiro MT, Braga C, de Souza WV, Marques ET, Cummings DAT. From re-emergence to hyperendemicity: the natural history of the dengue epidemic in Brazil. PLoS Negl Trop Dis. 2011;5(1):e935.

28. Wakimoto MD, Camacho LAB, Guaraldo L, Damasceno LS, Brasil P. Dengue in children: a systematic review of clinical and laboratory factors associated with severity. Expert Rev Anti-Infect Ther. 2015;13:1441-56.

29. Thomas L, Moravie V, Besnier F, Valentino R, Kaidomar S, Coquet LV, et al. Clinical presentation of dengue among patients admitted to the adult emergency department of a tertiary care hospital in Martinique: implications for triage, management, and reporting. Ann Emerg Med. 2012;59(1):42-50.

30. Kuo H-J, Lee I-K, Liu J-W. Analyses of clinical and laboratory characteristics of dengue adults at their hospital presentations based on the World Health Organization clinical-phase framework: Emphasizing risk of severe dengue in the elderly. J Microbiol Immunol Infect. 2018;51:740-8.

\section{Publisher's Note}

Springer Nature remains neutral with regard to jurisdictional claims in published maps and institutional affiliations. 